\title{
An Exact Test for the Equality of Intraclass Correlation Coefficients Under Unequal Family Sizes
}

\author{
Madhusudan Bhandary \\ Columbus State University, bhandary_madhusudan@columbusstate.edu
}

Koji Fujiwara

North Dakota State University, koji.fujiwara@ndsu.edu

Follow this and additional works at: http://digitalcommons.wayne.edu/jmasm

Part of the Applied Statistics Commons, Social and Behavioral Sciences Commons, and the Statistical Theory Commons

\section{Recommended Citation}

Bhandary, Madhusudan and Fujiwara, Koji (2011) "An Exact Test for the Equality of Intraclass Correlation Coefficients Under Unequal Family Sizes," Journal of Modern Applied Statistical Methods: Vol. 10 : Iss. 1 , Article 20.

DOI: $10.22237 /$ jmasm/ 1304223540

Available at: http://digitalcommons.wayne.edu/jmasm/vol10/iss1/20 


\title{
An Exact Test for the Equality of Intraclass Correlation Coefficients Under Unequal Family Sizes
}

\author{
Madhusudan Bhandary \\ Columbus State University, \\ Columbus, GA USA
}

\author{
Koji Fujiwara \\ North Dakota State University, \\ Fargo, ND USA
}

\begin{abstract}
An exact test for the equality of two intraclass correlation coefficients under unequal family sizes based on two independent multi-normal samples is proposed. This exact test consistently and reliably produced results superior to those of the Likelihood Ratio Test (LRT) and the large sample Z-test proposed by Young and Bhandary (1998). The test generally performed better in terms of power (for higher intraclass correlation values) for various combinations of intraclass correlation coefficient values and the exact test remained closer to the significance level under the null hypothesis compared to the other two tests. For small sample situations, sizes of the LRT and large-sample Z-tests are drastically higher than alpha-level, but the size of the exact test is close to the alpha-level. The proposed exact test is computationally simple and can be used for both small and large sample situations.
\end{abstract}

Key words: Likelihood ratio test, Z-test, F-test, intraclass correlation coefficient.

\section{Introduction}

The intraclass correlation coefficient $\rho$ is widely used to measure the degree of intrafamily resemblance with respect to characteristics such as blood pressure, cholesterol, weight, height, stature, lung capacity, etc. Several authors have studied statistical inference concerning $\rho$ based on single multinormal samples (Scheffe, 1959; Rao, 1973; Rosner, et al., 1977, 1979; Donner \& Bull, 1983; Srivastava, 1984; Konishi, 1985; Gokhale \& SenGupta, 1986; SenGupta, 1988; Velu \& Rao, 1990).

Donner and Bull (1983) discussed the likelihood ratio test (LRT) for testing the equality of two intraclass correlation coefficients based on two independent multinormal samples under equal family sizes. Konishi and Gupta (1987) proposed a modified LRT and derived its

Madhusudan Bhandary is an Associate Professor in the Department of Mathematics. Email him at: bhandary_madhusudan@columbusstate.edu. Koji Fujiwara is a graduate student in the Department of Statistics. Email him at: koji.fujiwara@ndsu.edu. asymptotic null distribution; they also discussed another test procedure based on a modification of Fisher's Z-transformation following Konishi (1985). Huang and Sinha (1993) considered an optimum invariant test for the equality of intraclass correlation coefficients under equal family sizes for more than two intraclass correlation coefficients based on independent samples from several multinormal distributions. For unequal family sizes, Young and Bhandary (1998) proposed a LRT, a large sample Z-test and a large sample $Z^{*}$-test for the equality of two intraclass correlation coefficients based on two independent multinormal samples.

For several populations and unequal family sizes, Bhandary and Alam (2000) proposed a LRT and a large sample ANOVA test for the equality of several intraclass correlation coefficients based on several independent multinormal samples. Donner and Zou (2002) proposed an asymptotic test for the equality of dependent intraclass correlation coefficients under unequal family sizes. Bhandary and Fujiwara (2006) proposed an Fmax test for several populations and under unequal family sizes. None of these studies, however, derived an exact test under unequal family sizes. It is an important practical problem to consider an exact test for the equality of 
intraclass correlation coefficients under unequal family sizes.

This article proposes an exact test for the equality of two intraclass correlation coefficients based on two independent multinormal samples under fixed but unequal family sizes. Developing an exact test for the equality of intraclass correlation coefficients under unequal family sizes would allow, for example, the determination of whether blood pressure, cholesterol, lung capacity, etc., among families in Native American or Caucasian races differ from the same among families in Asian races.

Tests of $H_{0}: \rho_{1}=\rho_{2}$ versus $H_{1}: \rho_{1} \neq \rho_{2}$ : Likelihood Ratio Test

$$
\text { Let } X_{i}=\left(x_{i 1}, x_{i 2}, \ldots, x_{i p_{i}}\right)^{\prime} \text { be a } p_{i} x 1
$$

vector of observations from $i^{\text {th }}$ family; $i=1,2, \ldots, k$. The structure of mean vector and the covariance matrix for the familial data is given by the following (Rao, 1973):

$$
\mu_{i}=\mu 1_{i}
$$

and

$$
\sum_{p_{i} x p_{i}}^{\Sigma_{i}}=\sigma^{2}\left(\begin{array}{cccc}
1 & \rho & \ldots & \rho \\
\rho & 1 & \ldots & \rho \\
\ldots & \ldots & \ldots & \ldots \\
\rho & \rho & \ldots & 1
\end{array}\right)
$$

where $1_{i}$ is a $p_{i} x 1$ vector of 1 's, $\mu(-\infty<\mu<\infty)$ is the common mean and $\sigma^{2}\left(\sigma^{2}>0\right)$ is the common variance of members of the family and $\rho$, which is called the intraclass correlation coefficient, is the coefficient of correlation among the members of the family and $\max _{1 \leq i \leq k}\left(-\frac{1}{p_{i}-1}\right) \leq \rho \leq 1$.

$$
\begin{array}{ccc}
\text { It } \quad \text { is } \quad \text { assumed } & \text { that } \\
x_{i} \sim N_{p_{i}}\left(\mu_{i}, \Sigma_{i}\right) ; i=1, \ldots, k, \quad \text { where } & N_{p_{i}}
\end{array}
$$

represents a $p_{i}$-variate normal distribution and $\mu_{i}, \Sigma_{i}{ }^{\prime} s$ are defined in (2.1). Let

$$
u_{i}=\left(u_{i 1}, u_{i 2}, \ldots, u_{i p_{i}}\right)^{\prime}=Q X_{i}
$$

where $Q$ is an orthogonal matrix. Under the orthogonal transformation (2.2), it can be observed that $u_{i} \sim N_{p_{i}}\left(\mu_{i}^{*}, \Sigma_{i}^{*}\right) ; i=1, \ldots, k$,

where

$$
\underset{\substack{p_{i} x 1}}{\mu_{i}^{*}}=(\mu, 0,0, \ldots, 0)^{\prime},
$$

and

$$
\Sigma_{i}^{*}=\sigma^{2}\left(\begin{array}{cccc}
\eta_{i} & 0 & \ldots & 0 \\
0 & 1-\rho & \ldots & 0 \\
\ldots & \ldots & \ldots & \ldots \\
0 & 0 & \ldots & 1-\rho
\end{array}\right),
$$

$$
\eta_{i}=p_{i}^{-1}\left\{1+\left(p_{i}-1\right) \rho\right\} .
$$

The transformation used on the data from $x$ to $u$ is independent of $\rho$ and can be accomplished using Helmert's orthogonal transformation.

Srivastava (1984) describes estimators of $\rho$ and $\sigma^{2}$ under unequal family sizes which are good substitutes for the maximum likelihood estimator and are given by the following:

$$
\hat{\rho}=1-\frac{\hat{\gamma}^{2}}{\hat{\sigma}^{2}}
$$

where 


$$
\begin{aligned}
& \hat{\sigma}^{2}=(k-1)^{-1} \sum_{i=1}^{k}\left(u_{i 1}-\hat{\mu}\right)^{2}+k^{-1} \hat{\gamma}^{2}\left(\sum_{i=1}^{k} a_{i}\right) \\
& \hat{\gamma}^{2}=\frac{\sum_{i=1}^{k} \sum_{r=2}^{p_{i}} u_{i r}^{2}}{\sum_{i=1}^{k}\left(p_{i}-1\right)} \\
& \hat{\mu}=k^{-1} \sum_{i=1}^{k} u_{i 1}
\end{aligned}
$$

and

$$
a_{i}=1-p_{i}^{-1}
$$

Next, consider the two sample problem with $\mathrm{k}_{1}$ and $\mathrm{k}_{2}$ families from each population. Let $x_{i}=\left(x_{i 1}, x_{i 2}, \ldots, x_{i p_{i}}\right)^{\prime}$ be a $p_{i} x 1$ vector of observations from $i^{\text {th }}$ family; $i=1, \ldots, k_{1}$ and $x_{i} \sim N_{p_{i}}\left(\mu_{1 i}, \Sigma_{1 i}\right)$, where

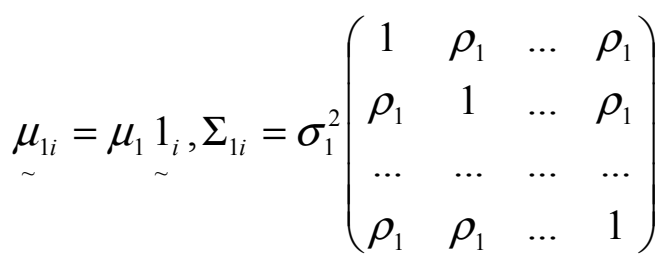

and $\max _{1 \leq i \leq k_{1}}\left(-\frac{1}{p_{i}-1}\right) \leq \rho_{1} \leq 1$.

Let $y_{j}=\left(y_{j 1}, y_{j 2}, \ldots, y_{j q_{j}}\right)^{\prime}$ be a $q_{j} x 1$

vector of observations from $\mathrm{j}^{\text {th }}$ family in the second population ; $\mathrm{j}=1, \ldots, \mathrm{k}_{2}$ and, the distribution of $y_{j}$ is same as $x_{i}$ in (2.4) except $\mu_{1}$ is replaced by $\mu_{2}, \sigma_{1}^{2}$ is replaced by $\sigma_{2}^{2}$ and $\rho_{1}$ is replaced by $\rho_{2}$ and $\max _{1 \leq j \leq k_{2}}\left(-\frac{1}{q_{j}-1}\right) \leq \rho_{2} \leq 1$ :

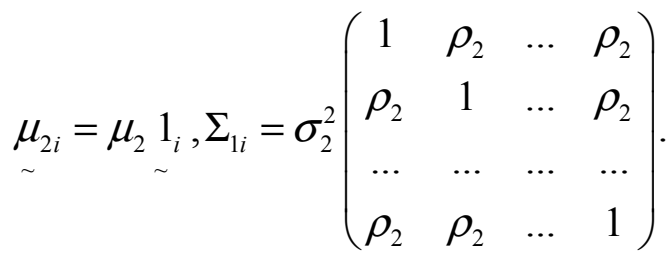

Using orthogonal transformation, the data vector can be transformed from $x_{i}$ to $u_{i}$ and $y_{j}$ to $v_{j}$ as follows:

$u_{i}=\left(u_{i 1}, u_{i 2}, \ldots, u_{i p_{i}}\right)^{\prime} \sim N_{p_{i}}\left(\mu_{1 i}^{*}, \Sigma_{1 i}^{*}\right) ; i=1, \ldots, k_{1}$

and

$v_{j}=\left(v_{j 1}, v_{j 2}, \ldots, v_{j q_{j}}\right)^{\prime} \sim N_{q_{j}}\left(\mu_{2 j}^{*}, \Sigma_{2 j}^{*}\right) ; j=1, \ldots, k_{2}$

where

$$
\begin{aligned}
& \mu_{1 i}^{*}=\left(\mu_{1}, 0,0, \ldots, 0\right)^{\prime}, \Sigma_{1 i}^{*}=\sigma_{1}^{2}\left(\begin{array}{cccc}
\eta_{i} & 0 & \ldots & 0 \\
0 & 1-\rho_{1} & \ldots & 0 \\
\ldots & \ldots & \ldots & \ldots \\
0 & 0 & \ldots & 1-\rho_{1}
\end{array}\right) \\
& \eta_{i}=p_{i}^{-1}\left\{1+\left(p_{i}-1\right) \rho_{1}\right\}
\end{aligned}
$$

$\mu_{2 j}^{*}$ and $\Sigma_{2 j}^{*}$ are the same as $\mu_{1 i}^{*}$ and $\Sigma_{1 i}^{*}$ respectively except that $\mu_{1}$ is replaced by $\mu_{2}$ ,$\sigma_{1}^{2}$ is replaced by $\sigma_{2}^{2}, \rho_{1}$ is replaced by $\rho_{2}$ and $\eta_{i}$ is replaced by $\xi_{j}=q_{j}^{-1}\left\{1+\left(q_{j}-1\right) \rho_{2}\right\}$. The transformations used on the data from $x$ to $u$ and $y$ to $v$ are independent of $\rho_{1}$ and $\rho_{2}$; note it is assumed that $\sigma_{1}^{2}=\sigma_{2}^{2}=\sigma^{2}$.

Under the above setup, Young and Bhandary (1998) derived likelihood ratio test 
statistic for testing $H_{0}: \rho_{1}=\rho_{2} \quad$ vs. $H_{1}: \rho_{1} \neq \rho_{2}$ which is given by the following:

$-2 \log \Lambda=\sum_{i=1}^{k_{1}} \log \left[p_{i}^{-1}\left\{1+\left(p_{i}-1\right) \hat{\rho}\right\}\right]+\sum_{i=1}^{k_{1}}\left(p_{i}-1\right) \log (1-\hat{\rho})$

$+\sum_{j=1}^{k_{2}} \log \left[q_{j}^{-1}\left\{1+\left(q_{j}-1\right) \hat{\rho}\right\}\right]+\sum_{j=1}^{k_{2}}\left(q_{j}-1\right) \log (1-\hat{\rho})$

$\left.+\sum_{j=1}^{k_{2}}\left\{q_{j}\left(v_{j 1}-\hat{\mu}_{2}\right)^{2} /\left[1+\left(q_{j}-1\right) \hat{\rho}\right]\right\}+\sum_{j=1}^{k_{2}} \sum_{s=2}^{q_{j}} v_{j s}^{2} /(1-\hat{\rho})\right]$

$\left.+\sum_{j=1}^{k_{2}}\left\{q_{j}\left(v_{j 1}-\hat{\mu}_{2}\right)^{2} /\left[1+\left(q_{j}-1\right) \hat{\rho}\right]\right\}+\sum_{j=1}^{k_{2}} \sum_{s=2}^{q_{j}} v_{j s}^{2} /(1-\hat{\rho})\right]$

$-\sum_{i=1}^{k_{1}} \log \left[p_{i}^{-1}\left\{1+\left(p_{i}-1\right) \hat{\rho}_{1}\right\}\right]-\sum_{i=1}^{k_{1}}\left(p_{i}-1\right) \log \left(1-\hat{\rho}_{1}\right)$

$-\sum_{j=1}^{k_{2}} \log \left[q_{j}^{-1}\left\{1+\left(q_{j}-1\right) \hat{\rho}_{2}\right\}\right]-\sum_{j=1}^{k_{2}}\left(q_{j}-1\right) \log \left(1-\hat{\rho}_{2}\right)$

$-\frac{1}{\hat{\sigma}^{2}}\left[\sum_{i=1}^{k_{1}}\left\{p_{i}\left(u_{i 1}-\hat{\mu}_{1}\right)^{2} /\left[1+\left(p_{i}-1\right) \hat{\rho}_{1}\right]\right\}+\sum_{i=1}^{k_{1}} \sum_{r=2}^{p_{i}} u_{i r}^{2} /\left(1-\hat{\rho}_{1}\right)\right.$

$\left.+\sum_{j=1}^{k_{2}}\left\{q_{j}\left(v_{j 1}-\hat{\mu}_{2}\right)^{2} /\left[1+\left(q_{j}-1\right) \hat{\rho}_{2}\right]\right\}+\sum_{j=1}^{k_{2}} \sum_{s=2}^{q_{j}} v_{j s}^{2} /\left(1-\hat{\rho}_{2}\right)\right]$

where, $\Lambda=$ likelihood ratio test statistic,

$$
\begin{gathered}
\hat{\rho}=1-\frac{\hat{\gamma}^{2}}{\hat{\sigma}^{* 2}}, \\
\hat{\gamma}^{2}=\frac{\sum_{i=1}^{k_{1}} \sum_{r=2}^{p_{i}} u_{i r}^{2}+\sum_{j=1}^{k_{2}} \sum_{s=2}^{q_{j}} v_{j s}^{2}}{\sum_{i=1}^{k_{1}}\left(p_{i}-1\right)+\sum_{j=1}^{k_{2}}\left(q_{j}-1\right)},
\end{gathered}
$$

and

$$
\begin{aligned}
\hat{\sigma}^{* 2}= & \left(k_{1}+k_{2}-1\right)^{-1}\left[\sum_{i=1}^{k_{1}}\left(u_{i 1}-\hat{\mu}_{z}\right)^{2}+\sum_{j=1}^{k_{2}}\left(v_{j 1}-\hat{\mu}_{z}\right)^{2}\right] \\
& +\left(k_{1}+k_{2}\right)^{-1} \hat{\gamma}^{2}\left(\sum_{i=1}^{k_{1}} a_{i}+\sum_{j=1}^{k_{2}} b_{j}\right)
\end{aligned}
$$

which equals the estimate of $\sigma^{2}$ under $H_{0}$,

$$
\begin{aligned}
& a_{i}=1-p_{i}^{-1} \\
& b_{j}=1-q_{j}^{-1}
\end{aligned}
$$

$$
\begin{gathered}
\hat{\mu}_{z}=\left(k_{1}+k_{2}\right)^{-1}\left[\sum_{i=1}^{k_{1}} u_{i 1}+\sum_{j=1}^{k_{2}} v_{j 1}\right] \\
\hat{\rho}_{1}=1-\frac{\hat{\gamma}_{1}^{2}}{\hat{\sigma}_{1}^{2}}
\end{gathered}
$$

where

$$
\begin{gathered}
\hat{\gamma}_{1}^{2}=\frac{\sum_{i=1}^{k_{1}} \sum_{r=2}^{p_{i}} u_{i r}^{2}}{\sum_{i=1}^{k_{1}}\left(p_{i}-1\right)}, \\
\hat{\sigma}_{1}^{2}=\left(k_{1}-1\right)^{-1} \sum_{i=1}^{k_{1}}\left(u_{i 1}-\hat{\mu}_{1}\right)^{2}+k_{1}^{-1} \hat{\gamma}_{1}^{2}\left(\sum_{i=1}^{k_{1}} a_{i}\right)
\end{gathered}
$$

and

$$
\hat{\rho}_{2}=1-\frac{\hat{\gamma}_{2}^{2}}{\hat{\sigma}_{2}^{2}}
$$

where

$$
\begin{gathered}
\hat{\gamma}_{2}^{2}=\frac{\sum_{j=1}^{k_{2}} \sum_{s=2}^{q_{j}} v_{j s}^{2}}{\sum_{j=1}^{k_{2}}\left(q_{j}-1\right)} \\
\hat{\sigma}_{2}^{2}=\left(k_{2}-1\right)^{-1} \sum_{j=1}^{k_{2}}\left(v_{j 1}-\hat{\mu}_{2}\right)^{2}+k_{2}^{-1} \hat{\gamma}_{2}^{2}\left(\sum_{j=1}^{k_{2}} b_{j}\right) \\
\hat{\sigma}^{2}=\frac{1}{2}\left(\hat{\sigma}_{1}^{2}+\hat{\sigma}_{2}^{2}\right)=\text { estimate of } \sigma^{2} \text { under } H_{1}
\end{gathered}
$$

$$
\begin{aligned}
& \hat{\mu}_{1}=k_{1}^{-1} \sum_{i=1}^{k_{1}} u_{i 1}, \\
& \hat{\mu}_{2}=k_{2}^{-1} \sum_{j=1}^{k_{2}} v_{j 1} .
\end{aligned}
$$

It is known from asymptotic theory that $-2 \log \Lambda$ has an asymptotic Chi-square distribution with 1 degree of freedom. Here (2.7) is not exact -2 (loglikelihood ratio) because Srivastava's (1984) estimator of parameters was substituted instead of exact likelihood estimator (which are not closed form in this situation). 


\section{EXACT TEST FOR THE EQUALITY OF INTRACLASS CORRELATION COEFFICIENTS}

However, Srivastava's (1984) estimators are strongly consistent and hence the asymptotic behavior of (2.7) may not be accurately as Chisquare distribution. It is a close approximation to the Chi-square distribution.

Young and Bhandary (1998) also proposed large sample Z-test as follows:

$$
Z=\frac{\hat{\rho}_{1}-\hat{\rho}_{2}}{S \sqrt{\frac{1}{k_{1}}+\frac{1}{k_{2}}}}
$$

where, $\hat{\rho}_{1}=$ estimator of $\rho_{1}$ from the first sample using Srivastava (1984), $\hat{\rho}_{2}=$ estimator of $\rho_{2}$ from the second sample using Srivastava (1984) and $S^{2}=$ pooled estimator of variance under $H_{0}$ using the formula given by Srivastava and Katapa (1986) as follows:

Var $=$

$2(1-\rho)^{2}\left\{(\bar{p}-1)^{-1}+c^{2}-2(1-\rho)(\bar{p}-1)^{-1} k^{-1}\left(\sum_{i=1}^{k} a_{i}\right)\right\}$

where, $k=$ number of families in the sample,

$$
\begin{aligned}
& \bar{p}=k^{-1} \sum_{i=1}^{k} p_{i} \\
& c^{2}=1-2(1-\rho)^{2} k^{-1} \sum_{i=1}^{k} a_{i}+(1-\rho)^{2}\left[k^{-1} \sum_{i=1}^{k} a_{i}+(\bar{p}-1)^{-1} \bar{a}^{2}\right] \\
& \bar{a}=k^{-1} \sum_{i=1}^{k} a_{i}
\end{aligned}
$$

and $a_{i}=1-p_{i}^{-1}$.

It is clear that under $H_{0}$, the test statistic $\mathrm{Z}$ given by (2.8) has an asymptotic $\mathrm{N}(0,1)$ distribution. The statistic $\mathrm{Z}$ in (2.8) under $H_{0}$ may not be exactly $\mathrm{N}(0,1)$ asymptotically, but it is a close approximation for large sample situation. A rough sketch of proof is as follows:

i. $\quad \hat{\rho}_{1} \sim N\left(\rho, V(\hat{\rho}) / k_{1}\right) \quad$ asymptotically under $H_{0}$ by Srivastava and Katapa (1986), where $V(\hat{\rho})$ is given by (2.9). (*) ii. Similarly, $\quad \hat{\rho}_{2} \sim N\left(\rho, V(\hat{\rho}) / k_{2}\right)$ asymptotically under $H_{0}$ by Srivastava and Katapa (1986), where $V(\hat{\rho})$ is given by (2.9). (**)

iii. The approximate asymptotic distribution of $\mathrm{Z}$ is obtained in $(2.8)$ as $\mathrm{N}(0,1)$ using $\left(^{*}\right)$ and $(* *)$, where $S^{2}$ is $V(\hat{\rho})$ with its estimate given by (2.9) where $\rho$ is replaced by $\hat{\rho}$ under $H_{0}$.

Young and Bhandary (1998) showed through simulation that the likelihood ratio test given by (2.7) consistently produced results superior to those of the large sample Z-test given by (2.8). It can be observed that likelihood ratio test given by (2.7) is computationally complex and is also used asymptotically, that is, when family sizes are large (at least 30). However, a researcher may have a situation in which only a small sample is available; thus, an exact F-test is proposed which is computationally simple and can be used for both small sample and large sample situations.

Proposed Exact Test

The new exact test is described as follows:

$$
F=\frac{\sum_{i=1}^{k_{1}} \sum_{r=2}^{p_{i}} u_{i r}^{2} /\left\{\sum_{i=1}^{k_{1}}\left(p_{i}-1\right)\right\}}{\sum_{j=1}^{k_{2}} \sum_{s=2}^{q_{j}} v_{j s}^{2} /\left\{\sum_{j=1}^{k_{2}}\left(q_{j}-1\right)\right\}}
$$

It can be shown using (2.6) that

$$
\frac{\sum_{i=1}^{k_{1}} \sum_{r=2}^{p_{i}} u_{i r}^{2}}{\sigma^{2}\left(1-\rho_{1}\right)} \sim \chi_{\sum_{i=1}^{k_{1}}\left(p_{i}-1\right)}^{2}
$$

and if the following replacements are made, $u_{i r}$ by $v_{j s}, k_{1}$ by $k_{2}, \mathrm{r}$ by s, $p_{i}$ by $q_{j}$ and $\rho_{1}$ 
by $\rho_{2}$, in (2.11) expression another Chi-square distribution results with

$$
\sum_{j=1}^{k_{2}}\left(q_{j}-1\right) \text { d.f. }
$$

where, $\chi_{n}^{2}$ denotes a Chi-square distribution with $\mathrm{n}$ degrees of freedom (d.f.).

Hence, under $H_{0}$, the exact distribution of the F-test statistic given by (2.10) is an Fdistribution with degrees of freedom $\sum_{i=1}^{k_{1}}\left(p_{i}-1\right)$ and $\sum_{j=1}^{k_{2}}\left(q_{j}-1\right)$ respectively (using (2.11) and (2.12)). Hence, the critical region (or rejection region) for testing $H_{0}: \rho_{1}=\rho_{2}$ vs. $H_{1}: \rho_{1} \neq \rho_{2}$ can be written as follows:

$$
\begin{aligned}
& F>F_{\frac{\alpha}{2} ; \sum_{i=1}^{k_{1}}\left(p_{i}-1\right), \sum_{j=1}^{k_{2}}\left(q_{j}-1\right)} \\
& \text { or } \\
& F<F_{1-\frac{\alpha}{2} ; \sum_{i=1}^{k_{1}}\left(p_{i}-1\right), \sum_{j=1}^{k_{2}}\left(q_{j}-1\right)}
\end{aligned}
$$

where $\mathrm{F}$ is the test statistic given by (2.10) and $F_{\gamma ; a, b}$ is the upper $100 \gamma \%$ point of an Fdistribution with degrees of freedom $a$ and $b$ respectively.

The exact power function of the F-test given by (2.10) can be derived as follows:

$$
\begin{aligned}
\varphi\left(\rho_{1}, \rho_{2}\right) & =P\left(F>\frac{1-\rho_{2}}{1-\rho_{1}} \cdot F_{\frac{\alpha}{2} ; \sum_{i=1}^{k_{1}}\left(p_{i}-1\right), \sum_{j=1}^{k_{2}}\left(q_{j}-1\right)}\right) \\
& +P\left(F<\frac{1-\rho_{2}}{1-\rho_{1}} \cdot F_{1-\frac{\alpha}{2} ; \sum_{i=1}^{k_{1}}\left(p_{i}-1\right), \sum_{j=1}^{k_{2}}\left(q_{j}-1\right)}\right)
\end{aligned}
$$

where

$$
F=\frac{\frac{\sum_{i=1}^{k_{1}} \sum_{r=2}^{p_{i}} u_{i r}^{2}}{\sigma^{2}\left(1-\rho_{1}\right)} / \sum_{i=1}^{k_{1}}\left(p_{i}-1\right)}{\frac{\sum_{j=1}^{k_{2}} \sum_{s=2}^{q_{j}} v_{j s}^{2}}{\sigma^{2}\left(1-\rho_{2}\right)} / \sum_{j=1}^{k_{2}}\left(q_{j}-1\right)} \sim
$$

F-distribution with degrees of freedom $\sum_{i=1}^{k_{1}}\left(p_{i}-1\right)$ and $\sum_{j=1}^{k_{2}}\left(q_{j}-1\right)$ respectively (using (2.11) and (2.12)) under $H_{1}$.

It can be observed that the test statistic $\mathrm{F}$ given by (2.10) is very simple to compute and that the distribution of $\mathrm{F}$ is exact and hence can be used for both small sample and large sample situations.

Simulation Study

Multivariate normal random vectors were generated using the $\mathrm{R}$ program in order to evaluate the power of the $F$ statistic as compared to the LRT statistic and Z-statistic. Five, 15 and 30 vectors of family data were created for each of the two populations. The family size distribution was truncated to maintain the family size at a minimum of 2 and a maximum of 15 siblings. Previous research in simulating family sizes (Rosner, et al., 1977; Srivastava \& Keen, 1988) determined the parameter setting for FORTRAN IMSL negative binomial subroutine with a mean $=2.86$ and a success probability $=$ 0.483 . In this study, the mean was set to equal 2.86 and theta equals 41.2552. All parameters were set the same for each population, except the values of $\rho_{1}$ and $\rho_{2}$ which took on all combinations possible over the range of values from 0.1 to 0.9 at increments of 0.1 .

The $\mathrm{R}$ program produced estimates of $\rho_{1}$ and $\rho_{2}$ along with the F statistic, the LRT statistic and the Z- statistic 3,000 times for each particular combination of population parameters $\left(\rho_{1}\right.$ and $\left.\rho_{2}\right)$. The frequency of rejection for each test statistic at $\alpha=0.05$ was noted and the proportion of rejections are reported in Table 1 for various combinations of $\rho_{1}$ and $\rho_{2}$. Table 2 shows the size comparison for the LRT statistic, 


\section{EXACT TEST FOR THE EQUALITY OF INTRACLASS CORRELATION COEFFICIENTS}

the $\mathrm{F}$ statistic and the $\mathrm{Z}$ statistic for various combinations of $\rho_{1}$ and $\rho_{2}$. Figures 1-4 present power estimates as well as size estimates for the three tests.

This study found that the exact F-test showed consistently better results for higher $\rho_{1}$ and $\rho_{2}$ values in power as well as in size compared to the LRT test and large sample Ztest. For the small sample situation, the LRT test and large sample Z-test have sizes drastically higher than alpha-level whereas the exact $F$ test is close to the alpha-level. Based on these results, the $\mathrm{F}$ test is strongly recommended for use in practice.

Table 1: Rejection Proportions for $\alpha=0.05$

\begin{tabular}{|c|c|c|c|c|c|c|c|c|c|c|}
\hline \multirow[b]{2}{*}{ rhol } & \multirow[b]{2}{*}{ rho2 } & \multicolumn{3}{|c|}{$K=5$} & \multicolumn{3}{|c|}{$K=15$} & \multicolumn{3}{|c|}{$\mathrm{K}=30$} \\
\hline & & LRT & $\mathrm{F}$ & Z & LRT & $\mathrm{F}$ & Z & LRT & F & Z \\
\hline 0.1 & 0.2 & 0.1947 & 0.0673 & 0.0123 & 0.1377 & 0.0600 & 0.0233 & 0.0910 & 0.0780 & 0.0447 \\
\hline 0.1 & 0.4 & 0.1660 & 0.0857 & 0.0607 & 0.2477 & 0.2103 & 0.1647 & 0.5423 & 0.4330 & 0.3633 \\
\hline 0.1 & 0.6 & 0.3873 & 0.2520 & 0.1413 & 0.7470 & 0.6923 & 0.5497 & 0.9723 & 0.9557 & 0.8717 \\
\hline 0.1 & 0.8 & 0.7067 & 0.7500 & 0.4257 & 0.9870 & 0.9943 & 0.9237 & 1.0000 & 1.0000 & 0.9987 \\
\hline 0.3 & 0.2 & 0.1930 & 0.0597 & 0.0383 & 0.0807 & 0.0700 & 0.0470 & 0.0953 & 0.0817 & 0.0650 \\
\hline 0.3 & 0.4 & 0.1347 & 0.0540 & 0.0493 & 0.0813 & 0.0783 & 0.0557 & 0.0997 & 0.1073 & 0.0667 \\
\hline 0.3 & 0.6 & 0.1803 & 0.1570 & 0.1467 & 0.3477 & 0.3630 & 0.2593 & 0.6667 & 0.6633 & 0.4803 \\
\hline 0.3 & 0.8 & 0.5410 & 0.5917 & 0.2763 & 0.8870 & 0.9147 & 0.7397 & 0.9990 & 1.0000 & 0.9600 \\
\hline 0.5 & 0.2 & 0.2093 & 0.1303 & 0.0977 & 0.3013 & 0.2847 & 0.2180 & 0.5863 & 0.5310 & 0.4213 \\
\hline 0.5 & 0.4 & 0.1113 & 0.0607 & 0.0723 & 0.0740 & 0.0880 & 0.0680 & 0.1033 & 0.1163 & 0.1010 \\
\hline 0.5 & 0.6 & 0.1013 & 0.0663 & 0.1190 & 0.0737 & 0.0843 & 0.0877 & 0.1367 & 0.1663 & 0.1037 \\
\hline 0.5 & 0.8 & 0.3403 & 0.3997 & 0.2493 & 0.6977 & 0.7747 & 0.4183 & 0.9327 & 0.9603 & 0.7173 \\
\hline 0.7 & 0.2 & 0.3910 & 0.3757 & 0.2557 & 0.8183 & 0.8247 & 0.6033 & 0.9867 & 0.9873 & 0.9080 \\
\hline 0.7 & 0.4 & 0.2647 & 0.2740 & 0.1717 & 0.5623 & 0.6080 & 0.3400 & 0.7983 & 0.8413 & 0.5783 \\
\hline 0.7 & 0.6 & 0.0877 & 0.0730 & 0.1320 & 0.1080 & 0.1303 & 0.1070 & 0.1813 & 0.2227 & 0.1327 \\
\hline 0.7 & 0.8 & 0.1107 & 0.1143 & 0.1523 & 0.1827 & 0.2610 & 0.1623 & 0.3373 & 0.4393 & 0.2277 \\
\hline 0.9 & 0.2 & 0.8723 & 0.9390 & 0.5140 & 0.9963 & 1.0000 & 0.9667 & 1.0000 & 1.0000 & 1.0000 \\
\hline 0.9 & 0.4 & 0.8527 & 0.9317 & 0.4453 & 0.9967 & 0.9993 & 0.8607 & 1.0000 & 1.0000 & 0.9983 \\
\hline 0.9 & 0.6 & 0.4703 & 0.5330 & 0.2457 & 0.9710 & 0.9897 & 0.6973 & 0.9983 & 1.0000 & 0.9277 \\
\hline 0.9 & 0.8 & 0.1680 & 0.1930 & 0.1797 & 0.3827 & 0.5087 & 0.2173 & 0.7263 & 0.8420 & 0.4337 \\
\hline
\end{tabular}


Table 2: Checking the Alpha Level $(\alpha=0.05)$

\begin{tabular}{|c|c|c|c|c|c|c|c|c|c|c|}
\hline & & \multicolumn{3}{|c|}{$\mathrm{K}=5$} & \multicolumn{3}{c|}{$\mathrm{K}=15$} & \multicolumn{3}{c|}{$\mathrm{K}=30$} \\
\cline { 3 - 11 } rho1 & rho0 & LRT & $\mathrm{F}$ & $\mathrm{Z}$ & LRT & $\mathrm{F}$ & $\mathrm{Z}$ & LRT & $\mathrm{F}$ & $\mathrm{Z}$ \\
\hline 0.1 & 0.1 & 0.1913 & 0.0490 & 0.0120 & 0.0943 & 0.0480 & 0.0157 & 0.0770 & 0.0497 & 0.0153 \\
\hline 0.2 & 0.2 & 0.2443 & 0.0543 & 0.0273 & 0.0757 & 0.0500 & 0.0220 & 0.0477 & 0.0490 & 0.0220 \\
\hline 0.3 & 0.3 & 0.1300 & 0.0467 & 0.0317 & 0.0497 & 0.0480 & 0.0383 & 0.0387 & 0.0503 & 0.0330 \\
\hline 0.4 & 0.4 & 0.1497 & 0.0503 & 0.0523 & 0.0403 & 0.0513 & 0.0457 & 0.0410 & 0.0470 & 0.0410 \\
\hline 0.5 & 0.5 & 0.1093 & 0.0550 & 0.0617 & 0.0420 & 0.0540 & 0.0540 & 0.0363 & 0.0487 & 0.0433 \\
\hline 0.6 & 0.6 & 0.0930 & 0.0520 & 0.0943 & 0.0367 & 0.0457 & 0.0633 & 0.0393 & 0.0540 & 0.0547 \\
\hline 0.7 & 0.7 & 0.0547 & 0.0470 & 0.1413 & 0.0363 & 0.0573 & 0.0760 & 0.0337 & 0.0473 & 0.0540 \\
\hline 0.8 & 0.8 & 0.0610 & 0.0537 & 0.1500 & 0.0290 & 0.0457 & 0.0827 & 0.0307 & 0.0533 & 0.0663 \\
\hline 0.9 & 0.9 & 0.0597 & 0.0480 & 0.1987 & 0.0230 & 0.0430 & 0.0853 & 0.0327 & 0.0557 & 0.0623 \\
\hline
\end{tabular}

Figure 1: Alpha Level $(\mathrm{k}=5$, alpha $=0.05)$

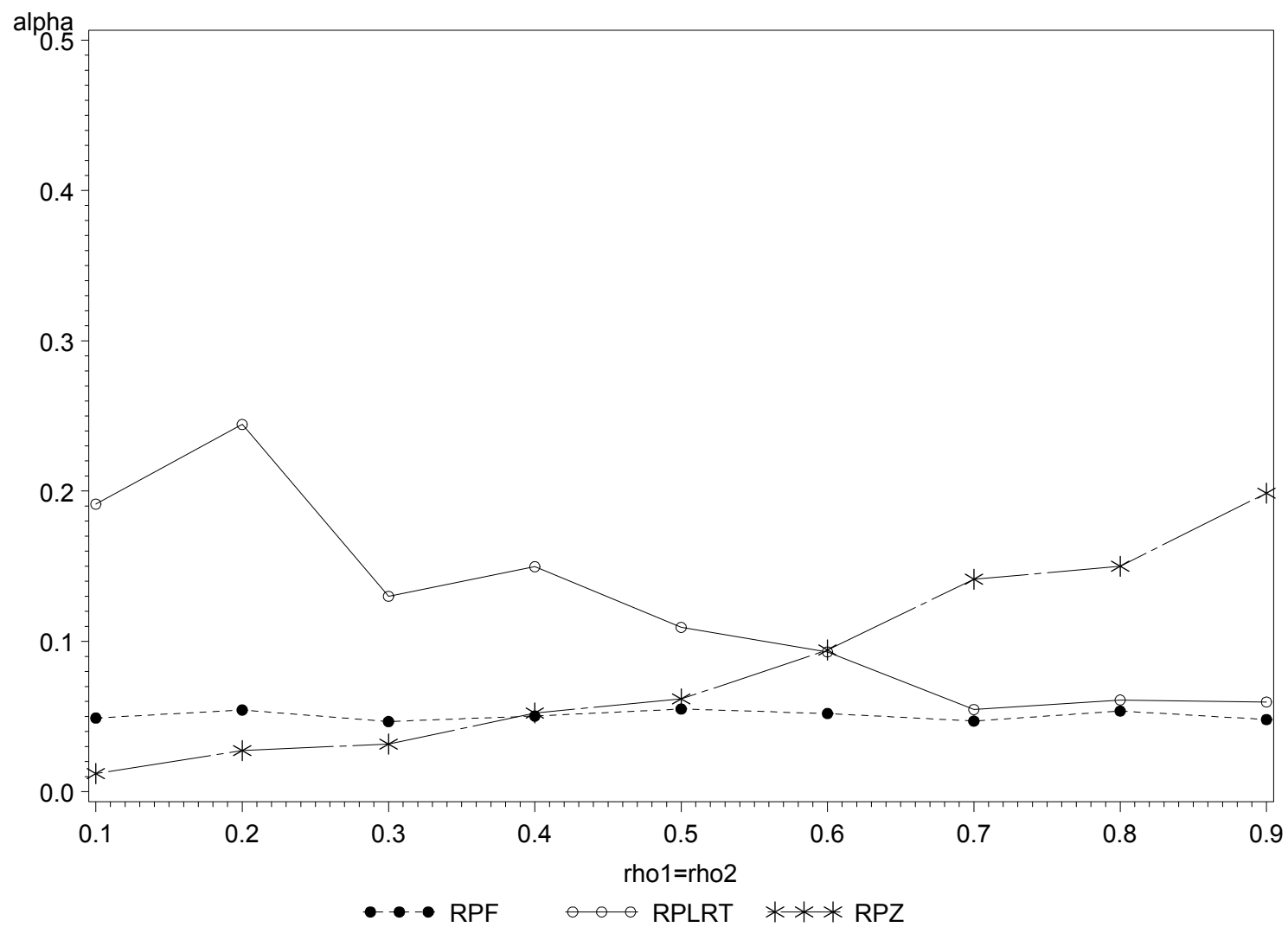


Figure 2: Alpha Level $(\mathrm{k}=15$, alpha $=0.05)$

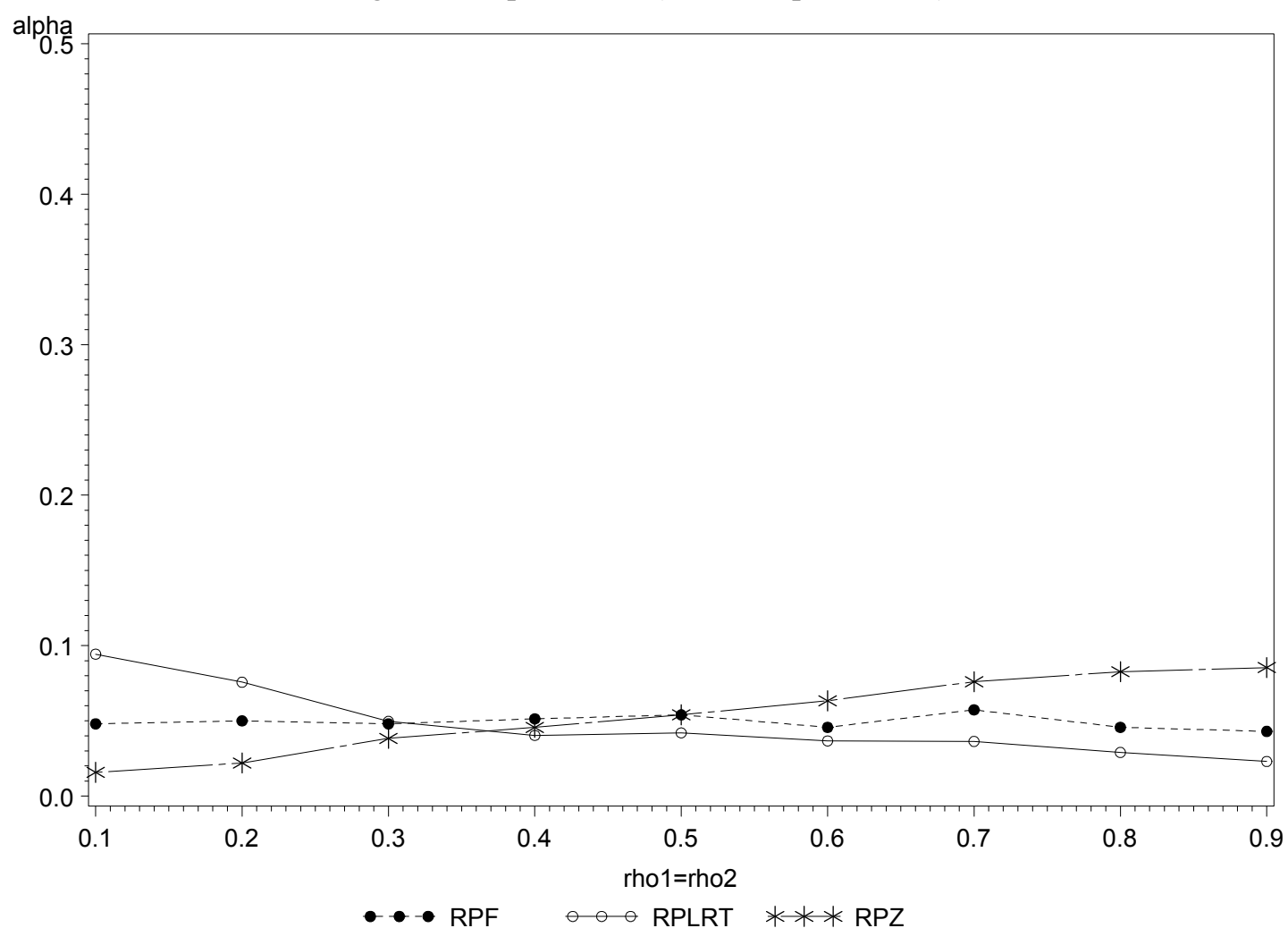

Figure 3: Power $($ rho1 $=0.9, \mathrm{k}=15$, alpha $=0.05)$

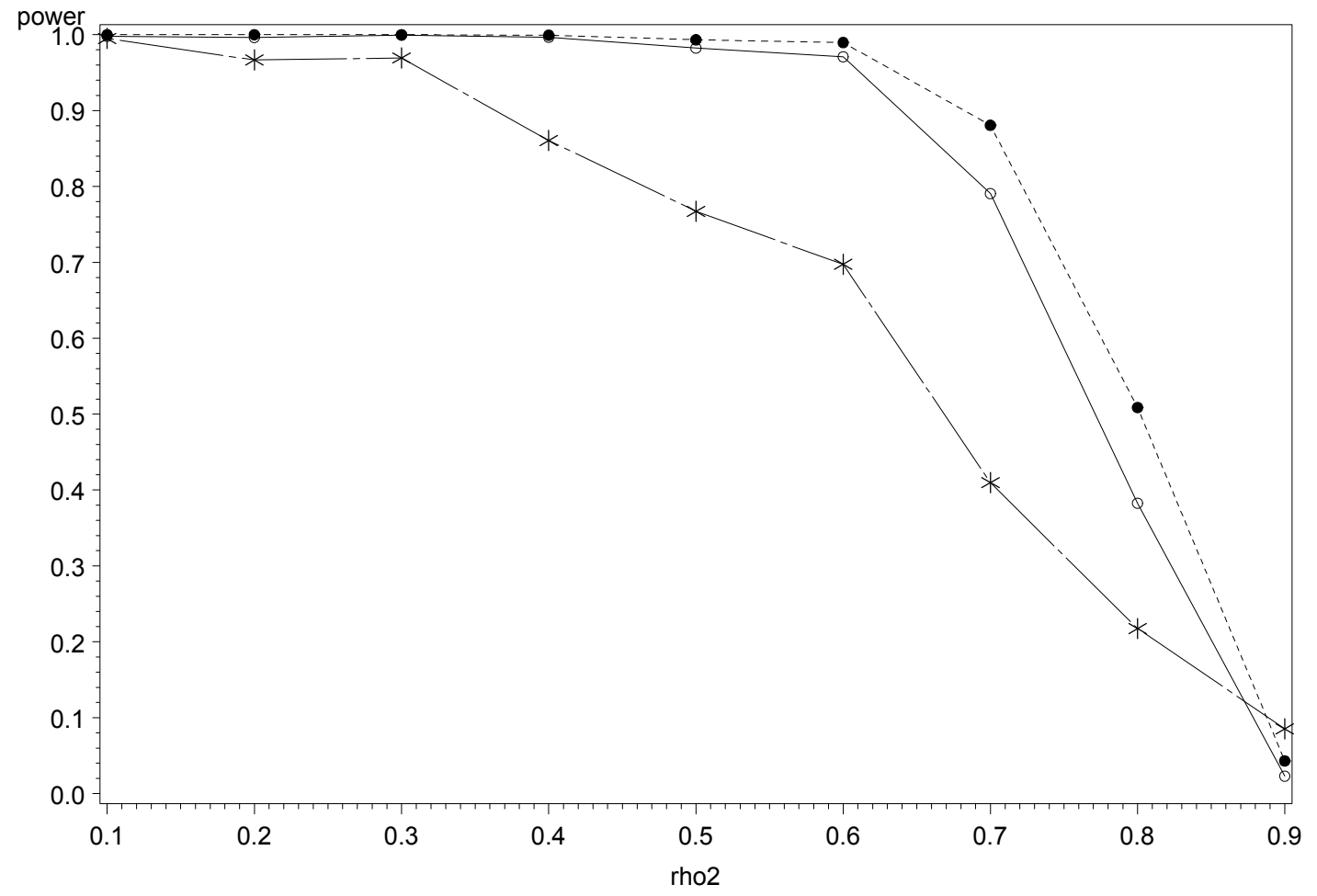




\section{BHANDARY \& FUJIWARA}

Figure 4: Power $(\mathrm{rho} 1=0.3, \mathrm{k}=30$, alpha $=0.05)$

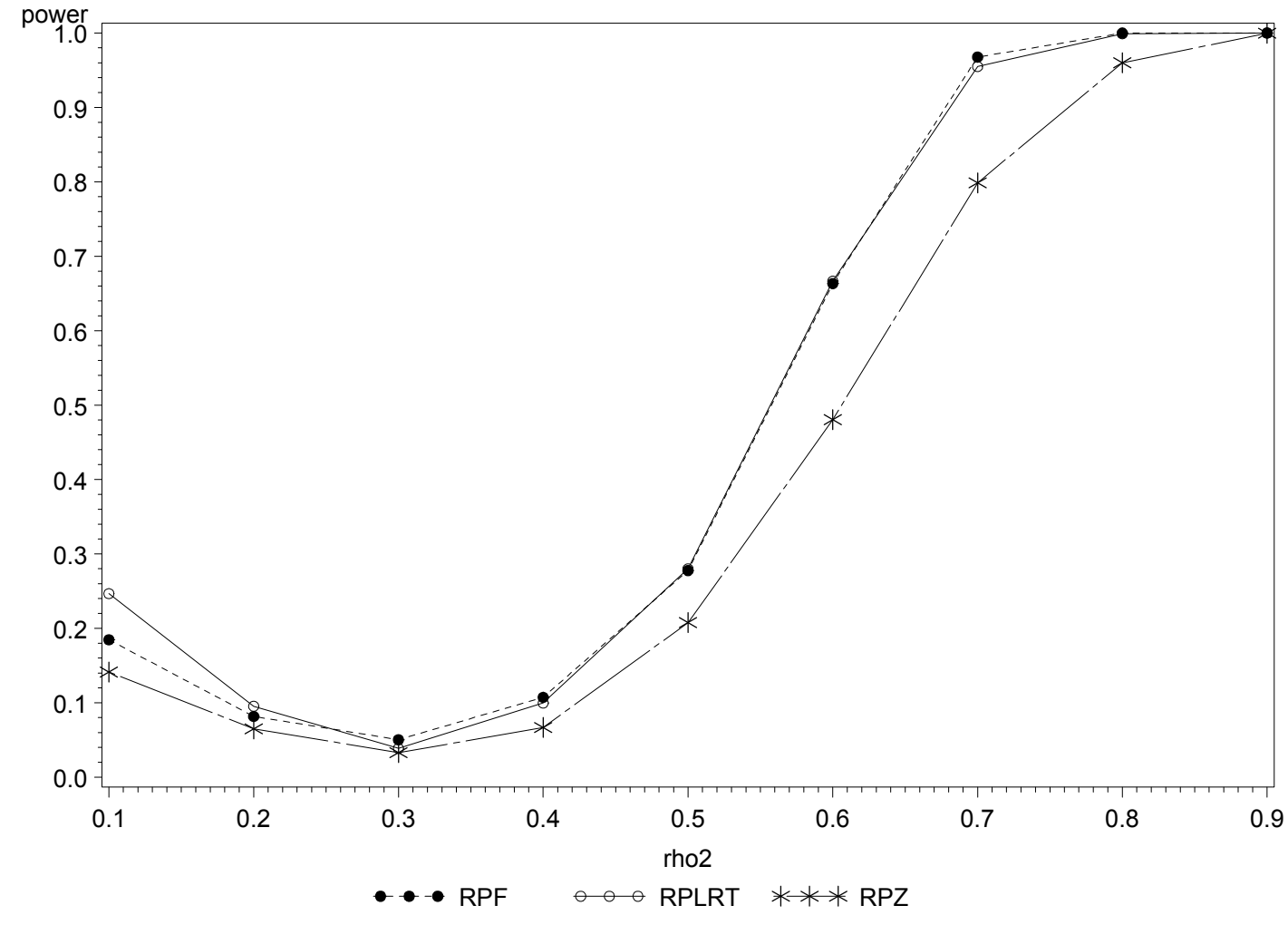

Example with Real Life Data

The main goal of this study is to find a better and easier way to compute the proposed exact F-test compared to the test given by Young and Bhandary (1998), the aim is not to find a test in situations such as $\sigma_{1}^{2}=\sigma_{2}^{2}$ and $\rho_{1} \neq \rho_{2}$ or $\sigma_{1}^{2} \neq \sigma_{2}^{2}$ and $\rho_{1}=\rho_{2}$ or for nonnormal data, etc. For this reason, the same example - values of pattern intensity on soles of feet in fourteen families - as used by Young and Bhandary (1998) is employed for this example. In this example two tests using real life data collected from Srivastava and Katapa (1986) are compared. First the data is randomly split into two samples as shown in Table 3.

First, the data is transformed by multiplying each observation vector by Helmert's orthogonal matrix Q, where

$$
\underset{p_{i} x p_{i}}{Q}=\left[\begin{array}{ccccc}
\frac{1}{\sqrt{p_{i}}} & \frac{1}{\sqrt{p_{i}}} & \frac{1}{\sqrt{p_{i}}} & \ldots & \frac{1}{\sqrt{p_{i}}} \\
\frac{1}{\sqrt{2}} & -\frac{1}{\sqrt{2}} & 0 & \ldots & 0 \\
\frac{1}{\sqrt{6}} & \frac{1}{\sqrt{6}} & -\frac{2}{\sqrt{6}} & 0 & 0 \\
\ldots & \ldots & \ldots & \ldots & \ldots \\
\frac{1}{\sqrt{p_{i}\left(p_{i}-1\right)}} & \frac{1}{\sqrt{p_{i}\left(p_{i}-1\right)}} & \frac{1}{\sqrt{p_{i}\left(p_{i}-1\right)}} & \ldots & -\frac{\left(p_{i}-1\right)}{\sqrt{p_{i}\left(p_{i}-1\right)}}
\end{array}\right]
$$

This results in transformed vectors $u_{i}$ and $v_{j}$ respectively for $i=1,2, \ldots, k_{1}$ and $j=1,2, \ldots, k_{2}$; here, $k_{1}=7$ and $k_{2}=7$.

Srivastava's formula given by (2.3) is used to compute intraclass correlation coefficients. The computed values of intraclass correlation coefficients are $\hat{\rho}_{1}=0.8708$, $\hat{\rho}_{2}=0.8544$ and $\hat{\rho}=0.85847$. The computed values of the LRT and F statistics are obtained 
Table 3: Random Sample Split for Values of Pattern Intensity on Soles of Feet in Fourteen Families Data

\begin{tabular}{|c|c|c|c|c|}
\hline Sample & Family\# & Mother & Father & Siblings \\
\hline \multirow{4}{*}{ A } & 12 & 2 & 4 & 2,4 \\
\cline { 2 - 5 } & 10 & 5 & 4 & $4,5,4$ \\
\cline { 2 - 5 } & 9 & 5 & 5 & 5,6 \\
\cline { 2 - 5 } & 1 & 2 & 3 & 2,2 \\
\cline { 2 - 5 } & 4 & 2 & 4 & $2,2,2,2,2$ \\
\cline { 2 - 5 } & 5 & 6 & 7 & 6,6 \\
\hline \multirow{4}{*}{ B } & 8 & 3 & 7 & $2,4,7,4,4,7,8$ \\
\cline { 2 - 5 } & 3 & 2 & 3 & $2,2,2$ \\
\cline { 2 - 5 } & 14 & 4 & 3 & $4,3,3$ \\
\cline { 2 - 5 } & 7 & 4 & 3 & $2,2,2$ \\
\cline { 2 - 5 } & 2 & 2 & 3 & $2,2,3,6,3,5,4$ \\
\cline { 2 - 5 } & 11 & 5 & 6 & 2,3 \\
\cline { 2 - 5 } & 13 & 6 & 3 & $4,3,3,3,4$ \\
\hline
\end{tabular}

from formula (2.7) and (2.10) respectively are as follows: $L R T$ statistic $=1.73374$ and $\mathrm{F}$ statistic $=2.15690$. Based on these results, the null hypothesis would not be rejected by either test at $1 \%, 5 \%$ or $10 \%$ levels. Intuitively, the test should not be rejected because the data is from one population and split into two samples. Considering the power and level of the two tests suggested in the simulation the proposed exact $\mathrm{F}$ test is recommended for use in practice.

\section{References}

Bhandary, M., \& Alam, M. K.(2000). Test for the equality of intraclass correlation coefficients under unequal family sizes for several populations. Communications in Statistics-Theory and Methods, 29(4), 755-768.
Bhandary, M., \& Fujiwara, K. (2006). A small sample test for the equality of intraclass correlation coefficients under unequal family sizes for several populations. Communications in Statistics-Simulation and Computation, 35(3), 765-778.

Donner, A., \& Bull, S. (1983). Inferences concerning a common intraclass correlation coefficient. Biometrics, 39, 771-775.

Donner, A., \& Zou, G. (2002). Testing the equality of dependent intraclass correlation coefficients. The Statistician, 51(3), 367-379.

Gokhale, D. V., \& SenGupta, A. (1986). Optimal tests for the correlation coefficient in a symmetric multivariate normal population. Journal of Statistical Planning and Inference, 14, 263-268.

Huang, W., \& Sinha, B. K. (1993). On optimum invariant tests of equality of intraclass correlation coefficients. Annals of the Institute of Statistical Mathematics, 45(3), 579-597. 


\section{BHANDARY \& FUJIWARA}

Konishi, S. (1985). Normalizing and variance stabilizing transformations for intraclass correlations. Annals of the Institute of Statistical Mathematics, 37, 87-94.

Konishi, S., \& Gupta, A. K. (1989). Testing the equality of several intraclass correlation coefficients. J Journal of Statistical Planning and Inference, 21, 93-105.

Rao, C. R. (1973). Linear statistical inference and its applications. New York: Wiley.

Rosner, B., Donner, A., \& Hennekens, C. H. (1977). Estimation of intraclass correlation from familial data. Applied Statistics, 26, 179187.

Rosner, B., Donner, A., \& Hennekens, C. H. (1979). Significance testing of interclass correlations from familial data. Biometrics, 35, 461-471.

SenGupta, A. (1988). On loss of power under additional information - an example. Scandinavian Journal of Statistics, 15, 25-31.
Scheffe, H. (1959). The analysis of variance. New York: Wiley.

Srivastava, M. S. (1984). Estimation of interclass correlations in familial data. Biometrika, 71, 177-185.

Srivastava, M. S., \& Katapa, R. S. (1986). Comparison of estimators of interclass and intraclass correlations from familial data. Canadian Journal of Statistics, 14, 29-42.

Srivastava, M. S., \& Keen, K. J. (1988). Estimation of the interclass correlation coefficient. Biometrika, 75, 731-739.

Velu, R., \& Rao, M. B. (1990). Estimation of parent-offspring correlation. Biometrika, 77(3), 557-562.

Young, D., \& Bhandary, M. (1998). Test for the equality of intraclass correlation coefficients under unequal family sizes. Biometrics, 54(4), 1363-1373. 